Table S1. Search strategies used for systematic review

\begin{tabular}{|c|c|c|}
\hline Database/Registry & $\begin{array}{l}\text { Search criteria }(09 / 05 / 2019- \\
09 / 08 / 2020)\end{array}$ & $\begin{array}{l}\text { Search criteria }(09 / 08 / 2020 \\
\text { only) }\end{array}$ \\
\hline Cochrane Library & $\begin{array}{l}\text { Search \#1: ascorbic acid AND diabetes } \\
\text { and limit to Trials tab; Search \#2: } \\
\text { "vitamin C" AND diabetes and limit to } \\
\text { Trials tab }\end{array}$ & - \\
\hline Scopus & $\begin{array}{l}\text { Search \#1: ascorb* AND diabetes AND } \\
\text { random*; Search \#2: "vitamin C" AND } \\
\text { diabetes AND random*; Search \#3: } \\
\text { ascorb* AND supplementation AND } \\
\text { diabetes; Search \#4: "vitamin C" AND } \\
\text { supplementation AND diabetes }\end{array}$ & $\begin{array}{l}\text { ('Ascorbic Acid'[Mesh] OR } \\
\text { ('Vitamin C') OR ('Vit C') } \\
\text { OR ascorb* OR } \\
\text { dehydroascorb*) AND } 1 . \\
\text { (MODY or NIDDM or } \\
\text { T2D*).[text words]. 2. (non } \\
\text { insulin* depend* or } \\
\text { noninsulin* depend* or } \\
\text { noninsulin?depend* or non } \\
\text { insulin?depend*).[text } \\
\text { words]. 3. (((late or adult* or } \\
\text { matur* or slow or stabl*) adj3 } \\
\text { onset) and diabet*).[text } \\
\text { words]. AND ((randomized } \\
\text { controlled trial[pt]) OR } \\
\text { (controlled clinical trial[pt]) } \\
\text { OR } \\
\text { (randomized[title/abstract] } \\
\text { OR } \\
\text { randomised[title/abstract]) } \\
\text { OR (placebo[title/abstract]) } \\
\text { OR (drug therapy[subject } \\
\text { heading]) OR } \\
\text { (randomly[title/abstract]) OR } \\
\text { (trial[title/abstract]) OR } \\
\text { (groups[title/abstract])) AND } \\
\text { NOT (animals) }\end{array}$ \\
\hline Embase & $\begin{array}{l}\text { Search \#1: 'ascorbic acid' AND diabetes } \\
\text { (and limit search to randomized } \\
\text { controlled trials only in the study } \\
\text { selection option; Search \#2: 'vitamin C' } \\
\text { AND diabetes (and limit search to } \\
\text { randomized controlled trials only in the } \\
\text { study selection option }\end{array}$ & As above \\
\hline $\begin{array}{l}\text { MEDLINE- } \\
\text { Pubmed }\end{array}$ & $\begin{array}{l}\text { Search \#1: ascorb* AND diabetes AND } \\
\text { random*; Search \#2: "vitamin C" AND } \\
\text { diabetes AND random*; Search \#3: } \\
\text { ascorb* AND supplementation AND } \\
\text { diabetes; Search \#4: "vitamin C" AND } \\
\text { supplementation AND diabetes }\end{array}$ & As above \\
\hline $\begin{array}{l}\text { Clinical trial } \\
\text { registries }\end{array}$ & $\begin{array}{l}\text { ClinicalTrials.gov: Search \#1: } \\
\text { condition: diabetes; other terms: }\end{array}$ & - \\
\hline
\end{tabular}




\begin{tabular}{|l|l|l|}
\hline ascorbic acid; Search \#2: condition: & \\
diabetes; other terms: vitamin C. & ANZCTR Search \#1: ascorbic acid; & \\
Search \#2: ascorb* AND diabetes. EU & Clinical Trial Register: Search \#1: & \\
& ascorbic acid; Search \#2: "vitamin C"; & \\
ISRCTN registry: Search \#1: ascorbic & acid AND diabetes; Search \#2: "vitamin & \\
C" AND diabetes & \\
\hline
\end{tabular}


Table S2: Sensitivity analyses

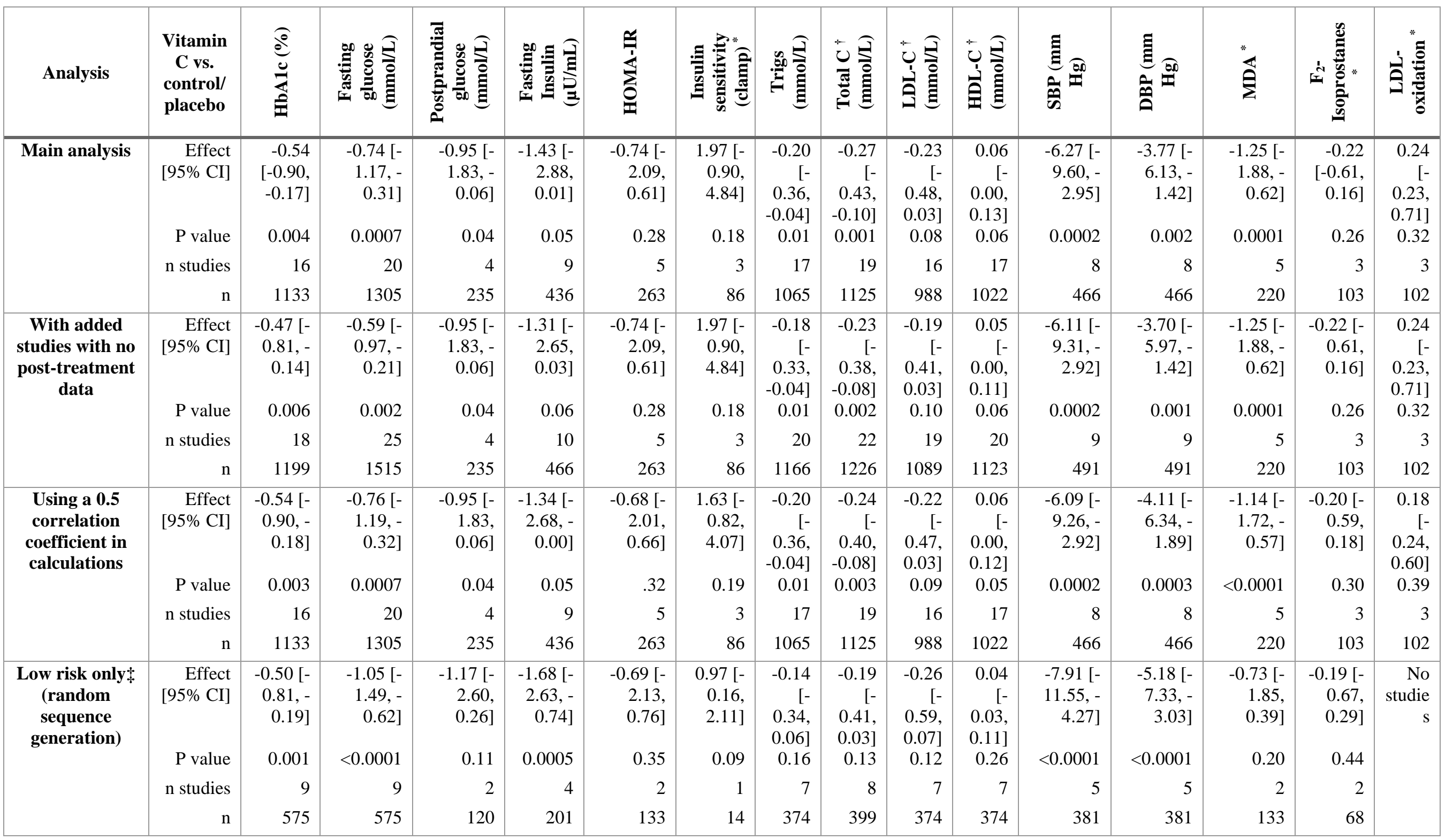




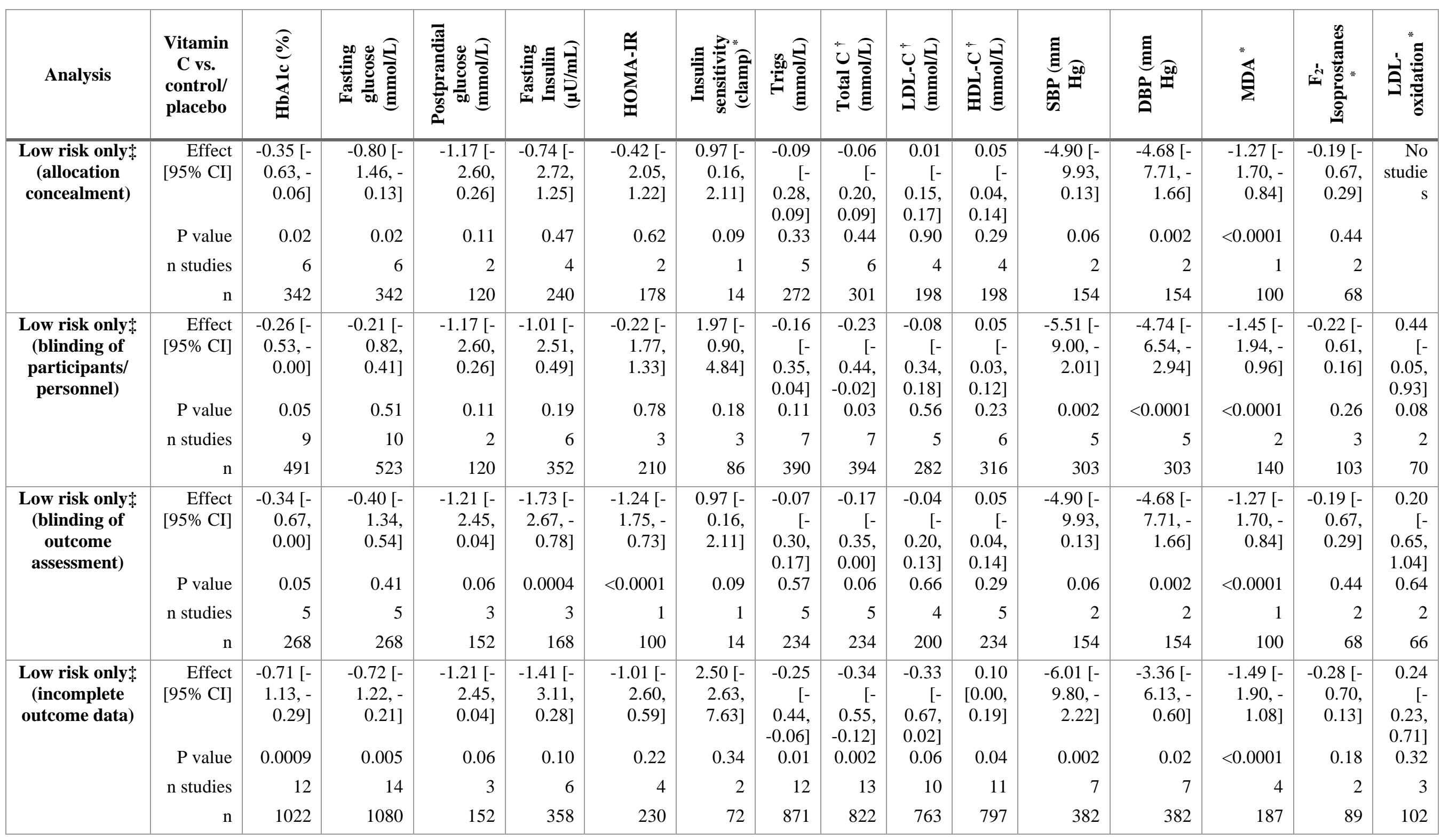




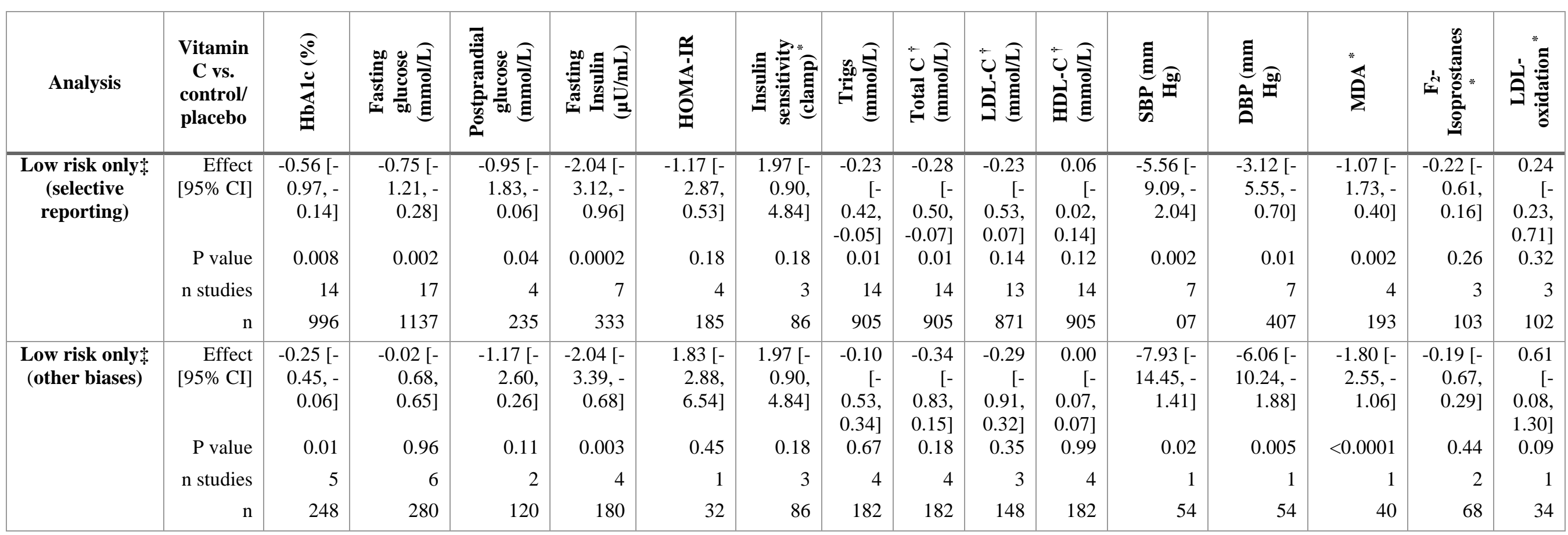

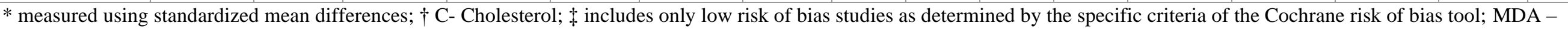
malondialdehyde. 
Table S3. Sub-group analyses of outcomes with > 10 studies: Mean difference of vitamin C supplementation compared to control

\begin{tabular}{|c|c|c|c|c|c|c|c|}
\hline Sub-group & Measures & HbA1c (\%) & $\begin{array}{c}\text { Fasting Glucose } \\
(\mathrm{mmol} / \mathrm{L})\end{array}$ & $\begin{array}{c}\text { Triglycerides } \\
(\mathrm{mmol} / \mathrm{L})\end{array}$ & $\begin{array}{c}\text { Total Cholesterol } \\
(\mathrm{mmol} / \mathrm{L})\end{array}$ & $\begin{array}{c}\text { LDL-Cholesterol } \\
(\mathrm{mmol} / \mathrm{L})\end{array}$ & $\begin{array}{c}\text { HDL-Cholesterol } \\
(\mathrm{mmol} / \mathrm{L})\end{array}$ \\
\hline \multicolumn{8}{|l|}{ Vitamin C dose } \\
\hline \multirow[t]{4}{*}{$<1000 \mathrm{mg} /$ day } & Effect $[95 \% \mathrm{CI}]$ & $-0.63[-1.41,0.15]$ & $-0.81[-1.57,-0.04]$ & $-0.31[-0.58,-0.05]$ & $-0.38[-0.62,-0.15]$ & $-0.55[-0.88,-0.22]$ & $0.09[-0.05,0.24]$ \\
\hline & $\mathrm{P}$ & 0.11 & 0.04 & 0.02 & $<0.01$ & 0.001 & 0.20 \\
\hline & n studies & 7 & 9 & 7 & 9 & 7 & 7 \\
\hline & $\mathrm{n}$ participants & 600 & 663 & 597 & 657 & 554 & 554 \\
\hline \multirow{5}{*}{$\geq 1000 \mathrm{mg} /$ day } & Effect $[95 \% \mathrm{CI}]$ & $-0.42[-0.68,-0.15]$ & $-0.69[-1.22,-0.16]$ & $-0.11[-0.30,0.08]$ & $-0.16[-0.39,0.07]$ & $0.03[-0.21,0.27]$ & $0.04[-0.01,0.09]$ \\
\hline & $\mathrm{P}$ & 0.002 & 0.01 & 0.25 & 0.17 & 0.81 & 0.09 \\
\hline & n studies & 9 & 11 & 10 & 10 & 9 & 10 \\
\hline & $\mathrm{n}$ participants & 533 & 642 & 468 & 468 & 434 & 468 \\
\hline & Sub-group $\mathrm{P}$ & 0.61 & 0.81 & 0.23 & 0.18 & 0.005 & 0.49 \\
\hline \multicolumn{8}{|l|}{ Trial duration } \\
\hline \multirow[t]{4}{*}{$<12$ weeks } & Effect $[95 \% \mathrm{CI}]$ & $-0.24[-0.64,0.17]$ & $-0.45[-1.18,0.28]$ & $-0.11[-0.21,0.00]$ & $-0.13[-0.26,-0.00]$ & $0.06[-0.16,0.28]$ & $0.04[-0.03,0.11]$ \\
\hline & $\mathrm{P}$ & 0.25 & 0.22 & 0.15 & 0.04 & 0.60 & 0.22 \\
\hline & n studies & 7 & 11 & 10 & 12 & 9 & 10 \\
\hline & $\mathrm{n}$ participants & 340 & 512 & 473 & 533 & 396 & 430 \\
\hline \multirow[t]{5}{*}{$\geq 12$ weeks } & Effect $[95 \% \mathrm{CI}]$ & $-0.79[-1.36,-0.23]$ & $-1.00[-1.53,-0.47]$ & $-0.35[-0.63,-0.08]$ & $-0.55[-0.91,-0.19]$ & $-0.55[-0.90,-0.21]$ & $0.10[-0.01,0.21]$ \\
\hline & $\mathrm{P}$ & 0.006 & 0.0002 & 0.01 & 0.003 & 0.002 & 0.06 \\
\hline & n studies & 9 & 9 & 7 & 7 & 7 & 7 \\
\hline & $\mathrm{n}$ participants & 793 & 793 & 592 & 592 & 592 & 592 \\
\hline & Sub-group $\mathrm{P}$ & 0.11 & 0.23 & 0.10 & 0.03 & 0.003 & 0.39 \\
\hline \multicolumn{8}{|l|}{ Baseline BMI } \\
\hline \multirow[t]{4}{*}{$<30.0 \mathrm{~kg} / \mathrm{m}^{2}$} & Effect $[95 \% \mathrm{CI}]$ & $-0.75[-1.25,-0.24]$ & $-0.90[-1.39,-0.41]$ & $-0.21[-0.39,-0.03]$ & $-0.22[-0.40,-0.05]$ & $-0.21[-0.52,0.10]$ & $0.09[0.01,0.17]$ \\
\hline & $\mathrm{P}$ & 0.004 & 0.0003 & 0.02 & 0.01 & 0.18 & 0.04 \\
\hline & n studies & 10 & 12 & 13 & 13 & 12 & 12 \\
\hline & $\mathrm{n}$ participants & 935 & 1044 & 964 & 718 & 890 & 890 \\
\hline \multirow[t]{5}{*}{$\geq 30.0 \mathrm{~kg} / \mathrm{m}^{2}$} & Effect $[95 \% \mathrm{CI}]$ & $-0.27[-0.91,0.37]$ & $-0.91[-2.41,0.59]$ & $-0.21[-0.51,0.09]$ & $-0.08[-0.32,0.16]$ & $-0.28[-0.83,0.27]$ & $-0.01[-0.17,0.14]$ \\
\hline & $\mathrm{P}$ & 0.41 & 0.24 & 0.18 & 0.51 & 0.32 & 0.85 \\
\hline & n studies & 3 & 4 & 3 & 4 & 3 & 3 \\
\hline & $\mathrm{n}$ participants & 67 & 99 & 67 & 92 & 67 & 67 \\
\hline & Sub-group $\mathrm{P}$ & 0.25 & 1.00 & 0.98 & 0.35 & 0.82 & 0.23 \\
\hline \multicolumn{8}{|l|}{ Baseline vitamin $C$} \\
\hline \multirow[t]{2}{*}{$<23 \mu \mathrm{mol} / \mathrm{L}$} & Effect $[95 \% \mathrm{CI}]$ & $-0.21[-0.42,0.00]$ & $0.45[-0.78,1.68]$ & $0.10[-0.55,0.75]$ & $-0.40[-0.83,0.03]$ & No studies & $0.00[-0.22,0.22]$ \\
\hline & $\mathrm{P}$ & 0.05 & 0.48 & 0.76 & 0.07 & & 1 \\
\hline
\end{tabular}




\begin{tabular}{|c|c|c|c|c|c|c|c|}
\hline Sub-group & Measures & HbA1c (\%) & $\begin{array}{l}\text { Fasting Glucose } \\
(\mathrm{mmol} / \mathrm{L})\end{array}$ & $\begin{array}{c}\text { Triglycerides } \\
(\mathbf{m m o l} / \mathbf{L})\end{array}$ & $\begin{array}{c}\text { Total Cholesterol } \\
(\mathrm{mmol} / \mathrm{L})\end{array}$ & $\begin{array}{c}\text { LDL-Cholesterol } \\
(\mathrm{mmol} / \mathrm{L})\end{array}$ & $\begin{array}{c}\text { HDL-Cholesterol } \\
(\mathrm{mmol} / \mathrm{L})\end{array}$ \\
\hline & n studies & 3 & 4 & 1 & 1 & & 1 \\
\hline & $\mathrm{n}$ participants & 131 & 163 & 34 & 34 & & 34 \\
\hline \multirow[t]{5}{*}{$\geq 23 \mu \mathrm{mol} / \mathrm{L}$} & Effect $[95 \% \mathrm{CI}]$ & $-0.29[-0.72,0.13]$ & $-0.64[-1.20,-0.08]$ & $-0.16[-0.58,0.26]$ & $-0.19[-0.54,0.16]$ & $-0.24[-0.71,0.24]$ & $0.00[-0.07,0.07]$ \\
\hline & $\mathrm{P}$ & 0.18 & 0.03 & 0.45 & 0.30 & 0.33 & 0.96 \\
\hline & n studies & 4 & 4 & 4 & 5 & 4 & 4 \\
\hline & $\mathrm{n}$ participants & 283 & 283 & 180 & 205 & 180 & 180 \\
\hline & Sub-group $\mathrm{P}$ & 0.74 & 0.12 & 0.51 & 0.45 & N/A & 0.99 \\
\hline \multicolumn{8}{|l|}{$\begin{array}{c}\text { Active control vs. } \\
\text { Placebo }\end{array}$} \\
\hline \multirow{4}{*}{$\begin{array}{l}\text { Active control } \\
\text { comparator }\end{array}$} & Effect $[95 \% \mathrm{CI}]$ & $-0.38[-0.82,0.05]$ & $-1.03[-1.87,-0.18]$ & $-0.08[-0.25,0.10]$ & $-0.09[-0.56,0.38]$ & $-0.05[-0.62,0.53]$ & $0.03[-0.10,0.18]$ \\
\hline & $P$ & 0.08 & 0.02 & 0.39 & 0.70 & 0.87 & 0.65 \\
\hline & n studies & 3 & 5 & 5 & 5 & 5 & 5 \\
\hline & $\mathrm{n}$ participants & 122 & 231 & 231 & 231 & 231 & 231 \\
\hline \multirow[t]{5}{*}{ Placebo comparator } & Effect $[95 \% \mathrm{CI}]$ & $-0.57[-0.99,-0.15]$ & $-0.64[-1.14,-0.14]$ & $-0.25[-0.44,-0.05]$ & $-0.31[-0.49,-0.14]$ & $-0.30[-0.60,0.00]$ & $0.08[-0.07,0.07]$ \\
\hline & $\mathrm{P}$ & 0.007 & 0.01 & 0.01 & 0.0004 & 0.05 & 0.06 \\
\hline & n studies & 13 & 15 & 12 & 14 & 11 & 12 \\
\hline & $\mathrm{n}$ participants & 1011 & 1074 & 834 & 894 & 757 & 791 \\
\hline & Sub-group P & 0.54 & 0.44 & 0.20 & 0.38 & 0.45 & 0.54 \\
\hline \multicolumn{8}{|l|}{$\begin{array}{c}\text { Lower vs higher } \\
\text { risk of bias }\end{array}$} \\
\hline \multirow{5}{*}{$\begin{array}{l}<4 \text { low risk of bias } \\
\text { Cochrane domains }\end{array}$} & Effect $[95 \% \mathrm{CI}]$ & $-0.64[-1.27,-0.02]$ & $-0.99[-1.52,-0.46]$ & $-0.22[-0.42,-0.01]$ & $-0.27[-0.47,-0.07]$ & $-0.22[-0.55,0.10]$ & $0.07[-0.02,0.16]$ \\
\hline & & & & & & & \\
\hline & $\mathrm{P}$ & 0.04 & 0.0002 & 0.04 & 0.008 & 0.17 & 0.13 \\
\hline & n studies & 10 & 13 & 12 & 14 & 12 & 12 \\
\hline & $\mathrm{n}$ participants & 785 & 925 & 783 & 843 & 740 & 740 \\
\hline \multirow{5}{*}{$\begin{array}{l}\geq 4 \text { low risk of bias } \\
\text { Cochrane domains }\end{array}$} & Effect $[95 \% \mathrm{CI}]$ & $-0.38[-0.70,-0.07]$ & $-0.25[-1.05,0.54]$ & $-0.15[-0.43,0.13]$ & $-0.28[-0.61,0.05]$ & $-0.14[-0.48,0.19]$ & $0.05[-0.22,0.22]$ \\
\hline & $\mathrm{P}$ & 0.03 & 0.53 & 0.29 & 0.10 & 0.41 & 028 \\
\hline & n studies & 6 & 7 & 5 & 5 & 4 & 5 \\
\hline & $\mathrm{n}$ participants & 348 & 380 & 282 & 282 & 248 & 282 \\
\hline & Sub-group $\mathrm{P}$ & 0.46 & 0.13 & 0.70 & 0.90 & 0.73 & 0.79 \\
\hline
\end{tabular}


Table S4. Meta-regression analyses of outcomes with $>10$ studies

\begin{tabular}{|c|c|c|c|c|c|c|}
\hline Modifying factor & HbA1c (\%) & $\begin{array}{l}\text { Fasting Glucose } \\
(\mathrm{mmol} / \mathrm{L})\end{array}$ & $\begin{array}{c}\text { Triglycerides } \\
(\mathrm{mmol} / \mathrm{L})\end{array}$ & $\begin{array}{c}\text { Total Cholesterol } \\
(\mathrm{mmol} / \mathrm{L})\end{array}$ & $\begin{array}{c}\text { LDL-Cholesterol } \\
(\mathrm{mmol} / \mathrm{L})\end{array}$ & $\begin{array}{c}\text { HDL-Cholesterol } \\
(\mathrm{mmol} / \mathrm{L})\end{array}$ \\
\hline \multicolumn{7}{|l|}{ Baseline HbAlc (\%) } \\
\hline$\beta$ & -0.4725 & -0.5768 & -0.1867 & -0.3239 & -0.5535 & 0.0874 \\
\hline$[95 \% \mathrm{CI}]$ & {$[-0.8595,-0.0855]$} & {$[-1.0463,-0.1075]$} & {$[-0.3539,-0.0195]$} & {$[-0.4773,-0.1704]$} & {$[-0.7609,-0.3460]$} & {$[-0.0051,0.1799]$} \\
\hline $\mathrm{P}$ & 0.017 & 0.016 & 0.029 & 0.000 & 0.005 & 0.064 \\
\hline n studies & 16 & 18 & 15 & 16 & 13 & 14 \\
\hline $\mathrm{I}^{2}$ (residual) & 83.65 & 71.90 & 60.77 & 36.29 & 52.22 & 72.56 \\
\hline \multicolumn{7}{|l|}{ Vitamin C dose (mg/day) } \\
\hline$\beta$ & 0.0001 & 0.0009 & 0.0001 & 0.0001 & 0.0007 & -0.0001 \\
\hline$[95 \% \mathrm{CI}]$ & {$[-0.0005,0.0008]$} & {$[0.0001,0.0017]$} & {$[-0.0001,0.0004]$} & {$[-0.0001,0.0004]$} & {$[0.0002,0.0030]$} & {$[-0.0002,0.0001]$} \\
\hline $\mathrm{P}$ & 0.661 & 0.020 & 0.303 & 0.352 & 0.013 & 0.319 \\
\hline n studies & 16 & 20 & 17 & 19 & 16 & 17 \\
\hline $\mathrm{I}^{2}$ (residual) & 88.76 & 71.84 & 73.02 & 63.06 & 79.12 & 67.79 \\
\hline \multicolumn{7}{|l|}{ Trial duration (days) } \\
\hline$\beta$ & -0.0062 & -0.0050 & -0.0026 & -0.0033 & -0.0036 & 0.0015 \\
\hline$[95 \% \mathrm{CI}]$ & {$[-0.0092,-0.0033]$} & {$[-0.0091,-0.0009]$} & {$[-0.0038,-0.0013]$} & {$[-0.0053,-0.0013]$} & {$[-0.0061,-0.0012]$} & {$[0.0008,0.0023]$} \\
\hline $\mathrm{P}$ & 0.000 & 0.016 & 0.000 & 0.001 & 0.004 & 0.000 \\
\hline n studies & 16 & 20 & 17 & 19 & 16 & 17 \\
\hline $\mathrm{I}^{2}$ (residual) & 74.12 & 66.23 & 41.33 & 45.22 & 74.43 & 50.06 \\
\hline \multicolumn{7}{|l|}{ Baseline Age (y) } \\
\hline$\beta$ & 0.0423 & 0.0434 & 0.0073 & 0.0138 & 0.0026 & -0.0118 \\
\hline$[95 \% \mathrm{CI}]$ & {$[-0.0043,0.0889]$} & {$[-0.0142,0.0949]$} & {$[-0.0136,0.0283]$} & {$[-0.0124,0.0400]$} & {$[-0.0337,0.0389]$} & {$[-0.0222,-0.0014]$} \\
\hline $\mathrm{P}$ & 0.075 & 0.147 & 0.492 & 0.303 & 0.889 & 0.026 \\
\hline n studies & 16 & 20 & 17 & 19 & 16 & 17 \\
\hline $\mathrm{I}^{2}$ (residual) & 86.04 & 72 & 74.54 & 60.92 & 83.46 & 65.74 \\
\hline \multicolumn{7}{|l|}{ Baseline BMI $\left(\mathrm{kg} / \mathrm{m}^{2}\right)$} \\
\hline$\beta$ & 0.1347 & 0.1102 & 0.0389 & 0.0637 & 0.0204 & -0.0440 \\
\hline$[95 \% \mathrm{CI}]$ & {$[-0.0055,0.2749]$} & {$[-0.0279,0.2482]$} & {$[-0.0290,0.1068]$} & {$[-0.0178,0.1452]$} & {$[-0.0915,0.1323]$} & {$[-0.0665,-0.0214]$} \\
\hline $\mathrm{P}$ & 0.060 & 0.118 & 0.262 & 0.125 & 0.721 & 0.000 \\
\hline n studies & 13 & 16 & 16 & 17 & 15 & 15 \\
\hline $\mathrm{I}^{2}$ (residual) & 83.98 & 67.59 & 72.93 & 63.42 & 85.58 & 43.98 \\
\hline \multicolumn{7}{|l|}{ Diabetes duration (y) } \\
\hline$\beta$ & 0.2108 & 0.1171 & 0.0168 & 0.0061 & 0.0204 & -0.0130 \\
\hline$[95 \% \mathrm{CI}]$ & {$[-0.0461,0.4677]$} & {$[-0.1140,0.3482]$} & {$[-0.0740,0.1077]$} & {$[-0.0778,0.0901]$} & {$[-0.0915,0.1323]$} & {$[-0.0455,0.0195]$} \\
\hline $\mathrm{P}$ & 0.108 & 0.321 & 0.717 & 0.886 & 0.721 & 0.432 \\
\hline
\end{tabular}




\begin{tabular}{|c|c|c|c|c|c|c|}
\hline Modifying factor & HbA1c (\%) & $\begin{array}{c}\text { Fasting Glucose } \\
(\mathrm{mmol} / \mathrm{L})\end{array}$ & $\begin{array}{c}\text { Triglycerides } \\
(\mathrm{mmol} / \mathrm{L})\end{array}$ & $\begin{array}{c}\text { Total Cholesterol } \\
(\mathrm{mmol} / \mathrm{L})\end{array}$ & $\begin{array}{c}\text { LDL-Cholesterol } \\
(\mathrm{mmol} / \mathrm{L})\end{array}$ & $\begin{array}{c}\text { HDL-Cholesterol } \\
(\mathrm{mmol} / \mathrm{L})\end{array}$ \\
\hline n studies & 10 & 13 & 13 & 14 & 12 & 13 \\
\hline $\mathrm{I}^{2}$ (residual) & 88.54 & 79 & 80.7 & 70.75 & 84.2 & 70.07 \\
\hline \multicolumn{7}{|l|}{ Sample size $(n)$} \\
\hline$\beta$ & -0.0091 & -0.0073 & -0.0034 & -0.0042 & -0.0045 & 0.0002 \\
\hline$[95 \% \mathrm{CI}]$ & {$[-0.0114,-0.0069]$} & {$[-0.0126,-0.0021]$} & {$[-0.0050,-0.0019]$} & {$[-0.0068,-0.0016]$} & {$[-0.0076,-0.0013]$} & {$[0.0011,0.0029]$} \\
\hline $\mathrm{P}$ & 0.000 & 0.006 & 0.000 & 0.001 & 0.005 & 0.000 \\
\hline n studies & 16 & 20 & 17 & 19 & 16 & 17 \\
\hline $\mathrm{I}^{2}$ (residual) & 33.92 & 60.26 & 39.24 & 47.37 & 75.15 & 46.62 \\
\hline \multicolumn{7}{|l|}{ Baseline vitamin $C(\mu \mathrm{mol} / \mathrm{L})$} \\
\hline$\beta$ & 0.0012 & -0.0058 & -0.0093 & 0.0055 & 0.0662 & 0.0018 \\
\hline$[95 \% \mathrm{CI}]$ & {$[-0.0160,0.0185]$} & {$[-0.0576,0.0461]$} & {$[-0.0521,0.0335]$} & {$[-0.0147,0.0256]$} & {$[-0.0715,0.2039]$} & {$[-0.0058,0.0094]$} \\
\hline $\mathrm{P}$ & 0.889 & 0.828 & 0.671 & 0.596 & 0.346 & 0.649 \\
\hline n studies & 7 & 8 & 5 & 6 & 4 & 5 \\
\hline $\mathrm{I}^{2}$ (residual) & 33.83 & 66.42 & 81.19 & 57.43 & 71.63 & 0 \\
\hline
\end{tabular}


Table S5. GRADE Evidence Profile for effect of vitamin C supplementation on glycemic control and cardiovascular risk factors in people with Type 2 diabetes

\begin{tabular}{|c|c|c|c|c|c|c|c|c|c|c|c|}
\hline \multicolumn{7}{|l|}{$\begin{array}{c}\text { Certainty } \\
\text { assessment }\end{array}$} & \multicolumn{2}{|l|}{$\begin{array}{c}\text { № of } \\
\text { patients }\end{array}$} & \multicolumn{2}{|l|}{ Effect } & \multirow[b]{2}{*}{ Importance } \\
\hline № of studies & $\begin{array}{l}\text { Study } \\
\text { design }\end{array}$ & $\begin{array}{l}\text { Risk of } \\
\text { bias }\end{array}$ & Inconsistency & Indirectness & Imprecision & $\begin{array}{c}\text { Other } \\
\text { considerations }\end{array}$ & Vitamin C & $\begin{array}{l}\text { Control/ } \\
\text { Placebo }\end{array}$ & $\begin{array}{l}\text { Absolute } \\
\text { (95\% CI) }\end{array}$ & Certainty & \\
\hline \multicolumn{12}{|l|}{ HbA1c (\%) } \\
\hline 16 & $\begin{array}{c}\text { randomised } \\
\text { trials }\end{array}$ & not serious * & serious $^{\dagger}$ & serious * & serious ${ }^{\S}$ & none & 570 & 563 & $\begin{array}{c}\text { MD } 0.54 \text { lower } \\
(0.90 \text { lower to } \\
0.17 \text { lower })\end{array}$ & $\begin{array}{l}\oplus \bigcirc \bigcirc \bigcirc \\
\text { VERY LOW }\end{array}$ & CRITICAL \\
\hline
\end{tabular}

\section{Fasting glucose}

$(\mathbf{m m o l} / \mathrm{L})$

\begin{tabular}{|c|c|c|c|c|c|c|c|c|c|c|c|}
\hline 20 & $\begin{array}{l}\text { randomised } \\
\text { trials }\end{array}$ & serious $\|$ & serious $^{\dagger}$ & serious ${ }^{*}$ & serious ${ }^{\S}$ & none & 670 & 635 & \begin{tabular}{|c|} 
MD 0.74 lower \\
(1.17 lower to \\
0.31 lower $)$
\end{tabular} & $\begin{array}{l}\oplus \bigcirc \bigcirc \bigcirc \\
\text { VERY LOW }\end{array}$ & CRITICAL \\
\hline
\end{tabular}

\section{Systolic blood}

pressure (mmHg)

\begin{tabular}{|c|c|c|c|c|c|c|c|c|c|c|c|}
\hline 8 & $\begin{array}{c}\text { randomised } \\
\text { trials }\end{array}$ & not serious & not serious & serious $*$ & not serious & none & 236 & 230 & $\begin{array}{c}\text { MD } 6.27 \text { lower } \\
\text { (9.6 lower to } \\
2.95 \text { lower) }\end{array}$ & $\begin{array}{c}\oplus \oplus \oplus \bigcirc \\
\text { MODERATE }\end{array}$ & CRITICAL \\
\hline
\end{tabular}

\section{Diastolic blood}

pressure (mmHg)

\begin{tabular}{|c|c|c|c|c|c|c|c|c|c|c|c|}
\hline 8 & $\begin{array}{c}\text { randomised } \\
\text { trials }\end{array}$ & not serious & serious $^{\dagger}$ & serious $*$ & serious ${ }^{\S}$ & none & 236 & 230 & $\begin{array}{c}\text { MD 3.77 lower } \\
\text { (6.13 lower to } \\
1.42 \text { lower })\end{array}$ & $\begin{array}{c}\oplus \bigcirc \bigcirc \bigcirc \\
\text { VERY LOW }\end{array}$ & CRITICAL \\
\hline \multicolumn{12}{|c|}{$\begin{array}{c}\text { Triglycerides } \\
\text { (mmol/L) }\end{array}$} \\
\hline 17 & $\begin{array}{c}\text { randomised } \\
\text { trials }\end{array}$ & serious $\|$ & serious $^{\dagger}$ & serious $*$ & serious \# & none & 559 & 506 & $\begin{array}{c}\text { MD 0.2 lower } \\
(0.36 \text { lower to } \\
0.04 \text { lower })\end{array}$ & $\begin{array}{c}\oplus \bigcirc \bigcirc \bigcirc \\
\text { VERY LOW }\end{array}$ & CRITICAL \\
\hline
\end{tabular}

Total cholesterol

(mmol/L) 


\begin{tabular}{|c|c|c|c|c|c|c|c|c|c|c|c|}
\hline \multicolumn{7}{|l|}{$\begin{array}{c}\text { Certainty } \\
\text { assessment }\end{array}$} & \multicolumn{2}{|l|}{$\begin{array}{c}\text { № of } \\
\text { patients }\end{array}$} & \multicolumn{2}{|l|}{ Effect } & \\
\hline № of studies & $\begin{array}{l}\text { Study } \\
\text { design }\end{array}$ & $\begin{array}{c}\text { Risk of } \\
\text { bias }\end{array}$ & Inconsistency & Indirectness & Imprecision & $\begin{array}{c}\text { Other } \\
\text { considerations }\end{array}$ & Vitamin $\mathrm{C}$ & $\begin{array}{l}\text { Control/ } \\
\text { Placebo }\end{array}$ & $\begin{array}{l}\text { Absolute } \\
(95 \% \text { CI) }\end{array}$ & Certainty & Importance \\
\hline 19 & $\begin{array}{l}\text { randomised } \\
\text { trials }\end{array}$ & serious \| & serious $^{\dagger}$ & serious ${ }^{\sharp}$ & serious \# & none & 589 & 536 & $\begin{array}{c}\text { MD } 0.27 \text { lower } \\
\text { (0.43 lower to } \\
0.1 \text { lower })\end{array}$ & $\begin{array}{l}\oplus \bigcirc \bigcirc \bigcirc \\
\text { VERY LOW }\end{array}$ & CRITICAL \\
\hline
\end{tabular}

\section{LDL Cholesterol}

(mmol/L)

\begin{tabular}{|c|c|c|c|c|c|c|c|c|c|c|c|}
\hline 16 & $\begin{array}{c}\text { randomised } \\
\text { trials }\end{array}$ & not serious & serious ${ }^{\dagger}$ & serious ${ }^{*}$ & serious \# & none & 522 & 466 & \begin{tabular}{|c} 
MD $\mathbf{0 . 2 3}$ lower \\
$(0.48$ lower to \\
0.03 higher $)$
\end{tabular} & $\begin{array}{l}\oplus \bigcirc \bigcirc \bigcirc \\
\text { VERY LOW }\end{array}$ & CRITICAL \\
\hline
\end{tabular}

HDL cholesterol

$(\mathrm{mmol} / \mathrm{L})$

\begin{tabular}{|c|c|c|c|c|c|c|c|c|c|c|c|}
\hline 17 & $\begin{array}{l}\text { randomised } \\
\text { trials }\end{array}$ & serious $\|$ & serious $^{\dagger}$ & serious ${ }^{\star}$ & serious \# & none & 539 & 483 & $\begin{array}{c}\text { MD 0.06 } \\
\text { higher } \\
\text { (0 to } 0.13 \\
\text { higher) }\end{array}$ & $\begin{array}{c}\oplus \bigcirc \bigcirc \bigcirc \\
\text { VERY LOW }\end{array}$ & CRITICAL \\
\hline
\end{tabular}

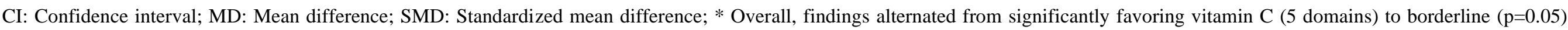

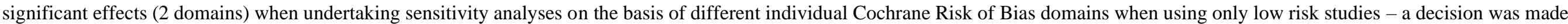

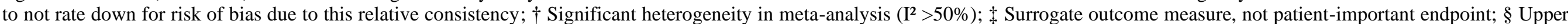

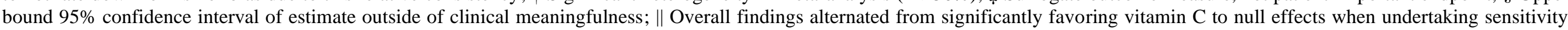

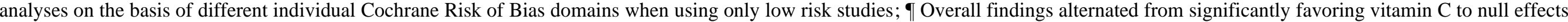

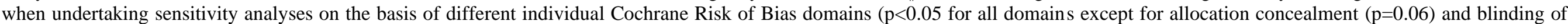

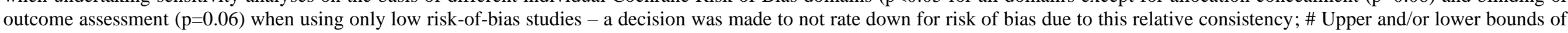
$95 \%$ confidence interval not clinically meaningful. 
Table S6. Adverse effects reported in included studies

\begin{tabular}{|c|c|c|}
\hline Study & Effects in Vitamin C group & Effects in Control group \\
\hline $\begin{array}{l}\text { Dakhale et al. } \\
2011(41)\end{array}$ & $\begin{array}{l}\text { No adverse effects on renal or liver } \\
\text { function }\end{array}$ & $\begin{array}{l}\text { No adverse effects on renal or liver } \\
\text { function }\end{array}$ \\
\hline $\begin{array}{l}\text { Devanandan et } \\
\text { al. } 2020(49)\end{array}$ & None reported & None reported \\
\hline $\begin{array}{l}\text { El-Aal et al. } \\
2018(50)\end{array}$ & $\begin{array}{l}\text { No adverse effects on renal or liver } \\
\text { function }\end{array}$ & $\begin{array}{l}\text { No adverse effects on renal or liver } \\
\text { function }\end{array}$ \\
\hline $\begin{array}{l}\text { Foroghi et al. } \\
2018(48)\end{array}$ & $\begin{array}{l}\text { Seven participants complained of } \\
\text { physical discomfort }\end{array}$ & None reported \\
\hline $\begin{array}{l}\text { Gillani et al. } \\
2017(18)\end{array}$ & $\begin{array}{l}23 \text { adverse events reported ( } 2 \\
\text { hypoglycemic episodes; } 3 \\
\text { hyperglycemic episodes; } 5 \text { wrong } \\
\text { timing of medication intake); no } \\
\text { adverse effects on renal or liver } \\
\text { function }\end{array}$ & $\begin{array}{l}89 \text { adverse events reported ( } 30 \\
\text { hypoglycemic episode; } 45 \\
\text { hyperglycemic episode; } 11 \text { wrong } \\
\text { timing of medication); no adverse } \\
\text { effects on renal or liver function }\end{array}$ \\
\hline $\begin{array}{l}\text { Kunsongkeit et } \\
\text { al. (46) }\end{array}$ & None reported & None reported \\
\hline $\begin{array}{l}\text { Mason et al. } \\
2016(42)\end{array}$ & $\begin{array}{l}\text { Minor GI discomfort during first } 1-2 \\
\text { weeks in one participant, after which } \\
\text { time it disappeared; no adverse } \\
\text { effects on renal or liver function }\end{array}$ & $\begin{array}{l}\text { No adverse effects on renal or liver } \\
\text { function }\end{array}$ \\
\hline $\begin{array}{l}\text { Mason et al. } \\
2019 \text { (5) }\end{array}$ & $\begin{array}{l}\text { No adverse effects on renal or liver } \\
\text { function }\end{array}$ & $\begin{array}{l}\text { A "feeling of depression" reported in } \\
\text { one participant; no adverse effects on } \\
\text { renal or liver function }\end{array}$ \\
\hline $\begin{array}{l}\text { Paolisso et al. } \\
1995(6)\end{array}$ & $\begin{array}{l}\text { No adverse effects on renal or liver } \\
\text { function }\end{array}$ & $\begin{array}{l}\text { No adverse effects on renal or liver } \\
\text { function }\end{array}$ \\
\hline $\begin{array}{l}\text { Ragheb et al. } \\
2020(59)\end{array}$ & $\begin{array}{l}\text { Survey Quality of Life scores } \\
\text { reported significantly better role } \\
\text { limitation to physical health and } \\
\text { emotional problems when compared } \\
\text { to control }\end{array}$ & ( \\
\hline $\begin{array}{l}\text { Rekha et al. } \\
2013 \text { (21) }\end{array}$ & $\begin{array}{l}\text { Some mild adverse effects such as } \\
\text { nausea, vomiting, diarrhea, } \\
\text { giddiness, headache, oral mucosal } \\
\text { erosion and fatigue }\end{array}$ & $\begin{array}{l}\text { Some mild adverse effects such as } \\
\text { nausea, vomiting, diarrhea, giddiness, } \\
\text { headache, oral mucosal erosion and } \\
\text { fatigue }\end{array}$ \\
\hline $\begin{array}{l}\text { Sanguanwong } \\
\text { et al. } 2016 \\
(20)\end{array}$ & None reported & None reported \\
\hline $\begin{array}{l}\text { Siavash et al. } \\
2014(47)\end{array}$ & Measured, but not reported & Measured, but not reported \\
\hline
\end{tabular}




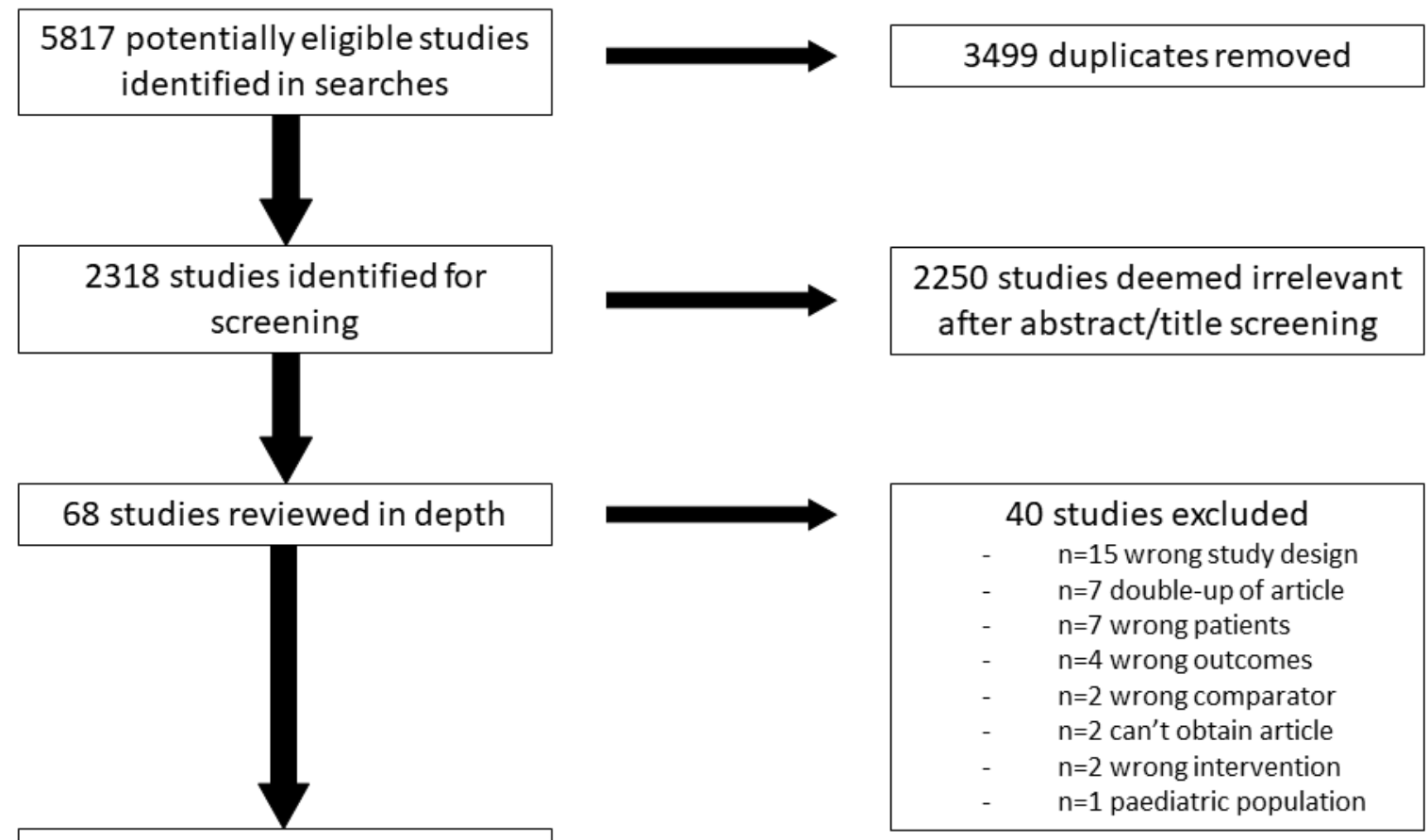

Figure S1. Selection of studies in the review 


\begin{tabular}{|c|c|c|c|c|c|c|c|}
\hline & 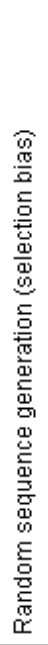 & 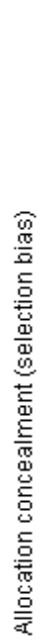 & 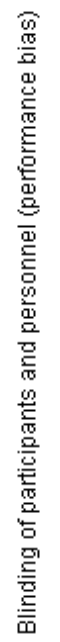 & 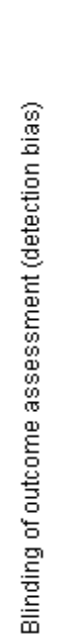 & 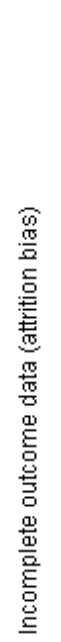 & 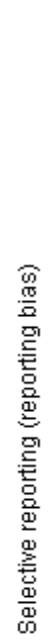 & $\begin{array}{l}\frac{0}{0} \\
\frac{\pi}{5} \\
\frac{2}{2} \\
\stackrel{5}{5}\end{array}$ \\
\hline Bhatt 2012 & $\odot$ & $\odot$ & 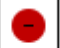 & $?$ & $\odot$ & $?$ & $?$ \\
\hline Chen 2006 & $?$ & $?$ & + & $?$ & $\bullet$ & $\odot$ & $\odot$ \\
\hline Dakhale 2011 & $\odot$ & $\odot$ & + & + & + & + & + \\
\hline Darko 2002 & $?$ & $?$ & + & $?$ & + & + & $?$ \\
\hline Devanandan 2020 & $\odot$ & $?$ & $?$ & $?$ & + & + & $?$ \\
\hline El Aal 2018 & $?$ & $?$ & $\theta$ & $?$ & + & + & $?$ \\
\hline Foroghi 2018 & $?$ & + & + & $?$ & + & $?$ & $?$ \\
\hline Ghaffari 2015 & $?$ & $?$ & $?$ & $?$ & $?$ & $?$ & $?$ \\
\hline Gillani 2017 & $?$ & $?$ & - & $?$ & + & $\odot$ & $\odot$ \\
\hline Gutierrez 2013 & $?$ & $?$ & - & + & + & + & $?$ \\
\hline Kunsongkeit 2019 & $?$ & $\odot$ & + & $?$ & + & $\odot$ & $?$ \\
\hline Lu 2005 & $?$ & $?$ & + & 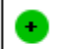 & $\odot$ & $\odot$ & 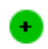 \\
\hline Mahmoudabadi 2011 & $?$ & $?$ & + & $?$ & $?$ & + & $?$ \\
\hline Mason 2016 & $\odot$ & 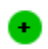 & + & 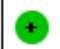 & $?$ & $\odot$ & 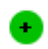 \\
\hline Mason 2019 & $\odot$ & 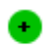 & + & 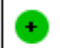 & 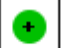 & $\odot$ & + \\
\hline Mazloom 2011 & $?$ & $?$ & 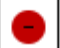 & $?$ & + & $?$ & $?$ \\
\hline Mullan 2002 & $?$ & $?$ & + & $?$ & + & + & $?$ \\
\hline Paolisso 1995 & $?$ & $?$ & + & $?$ & + & + & + \\
\hline Rafighi 2013 & 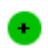 & $?$ & $?$ & $?$ & + & $\odot$ & $?$ \\
\hline Ragheb 2020 & $\odot$ & $?$ & - & $?$ & $?$ & $\odot$ & $?$ \\
\hline Rekha 2013 & $?$ & $?$ & 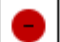 & - & $?$ & + & $?$ \\
\hline Sanguanwong 2016 & $\odot$ & $\odot$ & $\odot$ & 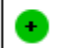 & $\odot$ & $\odot$ & $?$ \\
\hline ShakouriMahmoudabadi 2014 & $?$ & $?$ & + & $?$ & + & $\odot$ & + \\
\hline Shateri 2016 & $\odot$ & $?$ & + & $?$ & $?$ & + & $?$ \\
\hline Siavash 2014 & 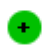 & 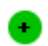 & $\Theta$ & $?$ & $?$ & $\odot$ & $?$ \\
\hline Tessier 2009 & $?$ & $?$ & + & $?$ & + & $\odot$ & $?$ \\
\hline Tousoulis 2007 & $?$ & $?$ & $O$ & $?$ & + & $\odot$ & $?$ \\
\hline Upritchard 2000 & 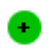 & 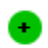 & $O$ & $?$ & + & $?$ & $?$ \\
\hline
\end{tabular}

Figure S2. Risk of bias assessment of included studies. Green with a "+" indicates "low risk" of bias; yellow with a "?" indicates "unclear risk" of bias; red with a "_. indicates "high risk” of bias 


\section{A}

Postprandial Glucose (mmol/L)

Vitamin C Control/Placebo

Mean Difference Weight

Study

$\mathrm{N} \quad \mathrm{N}$

with $95 \% \mathrm{Cl}$

(\%)

Dakhale 2011

Gutierrez 2013

33

$\mathrm{N}$

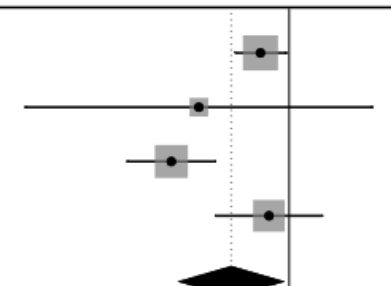

$-0.47[-0.89,-0.05] 34.68$

Mason 2019

24

33

8

Rekha 2013

27

27

55

28

Overall

139

96

95\% Prediction interval

Heterogeneity: $\mathrm{T}^{2}=0.54, \mathrm{I}^{2}=77.07 \%$

Test of $\theta_{\mathrm{i}}=\theta_{\mathrm{j}}: \mathrm{Q}(3)=13.08, \mathrm{p}=0.004$

Test of $\theta=0: z=-2.10, p=0.04$

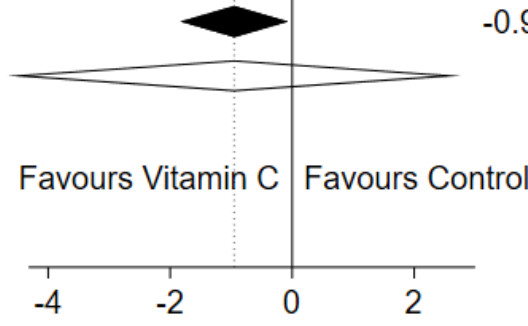

B

Fasting insulin $(\mu \mathrm{U} / \mathrm{mL})$

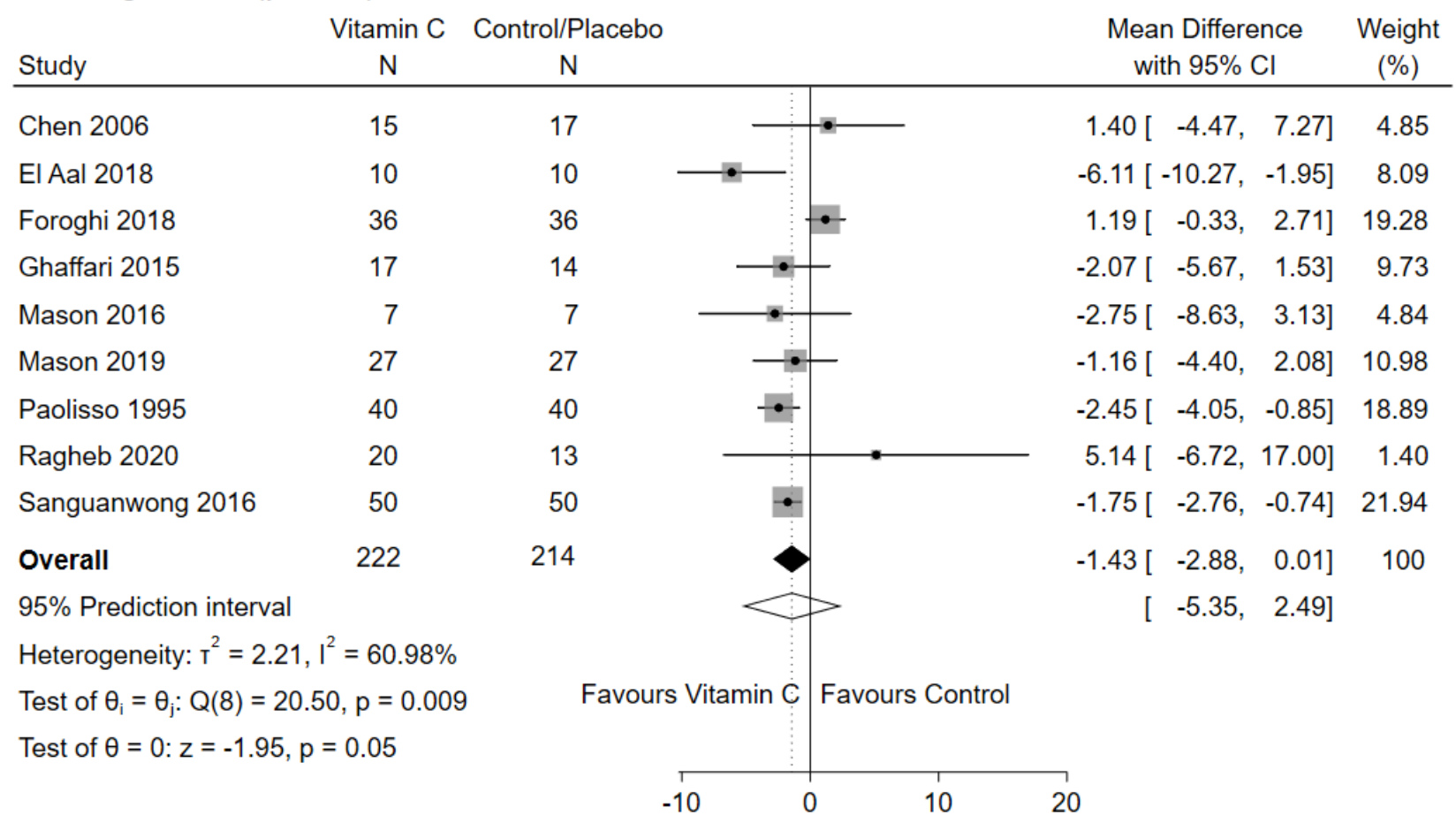

C 
HOMA-IR

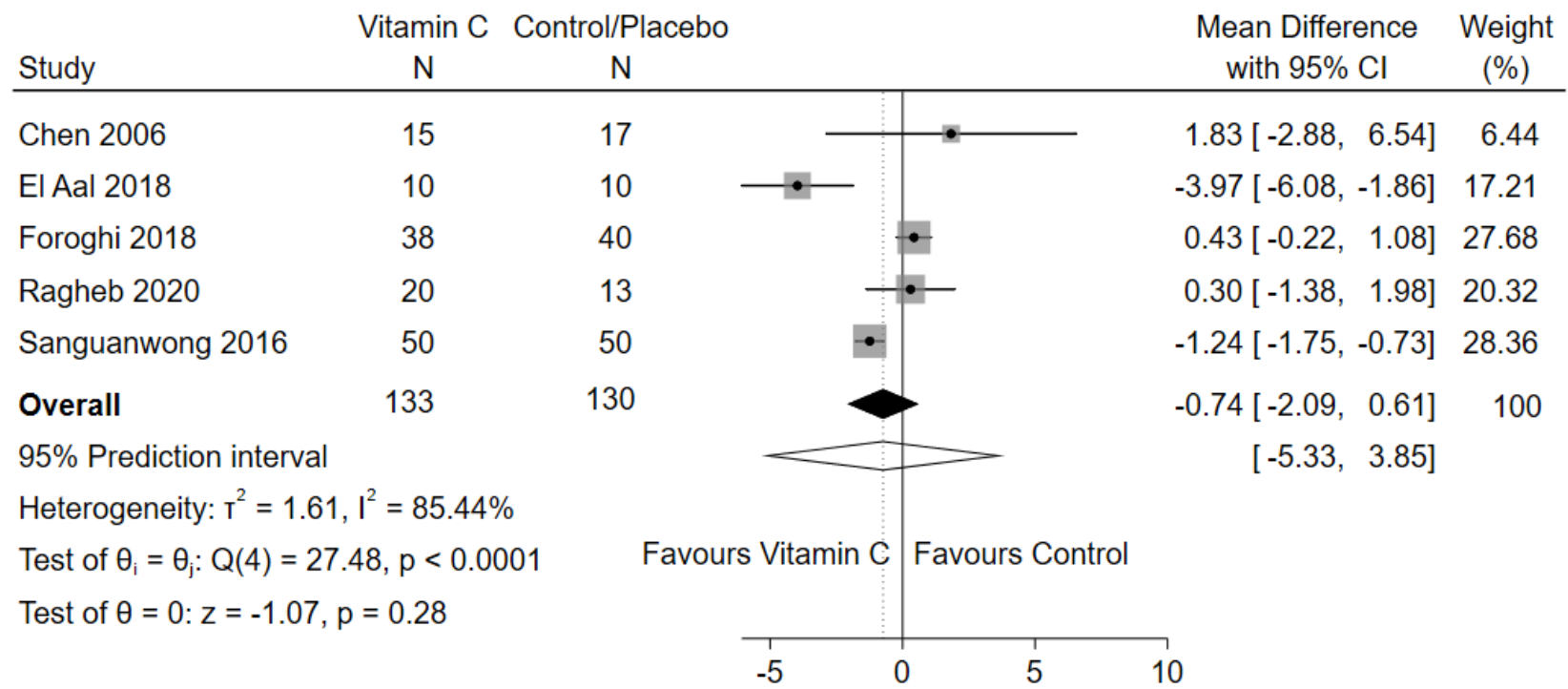

D

Insulin sensitivity (Clamp)

\begin{tabular}{|c|c|c|c|c|c|c|}
\hline Study & $\begin{array}{l}\text { Vitamin C Cor } \\
\qquad \mathrm{N}\end{array}$ & $\begin{array}{l}\mathrm{ol} / \mathrm{P} \\
\mathrm{N}\end{array}$ & & & $\begin{array}{c}\text { Standardized Mean Difference } \\
\text { with } 95 \% \mathrm{Cl}\end{array}$ & $\begin{array}{c}\text { Weight } \\
(\%)\end{array}$ \\
\hline Chen 2006 & 15 & 17 & & $\bullet$ & $-0.09\left[\begin{array}{ll}-0.78, & 0.61]\end{array}\right]$ & 34.29 \\
\hline Mason 2016 & 7 & 7 & & - & $0.97[-0.16,2.11]$ & 33.19 \\
\hline Paolisso 1995 & 20 & 20 & & $\bullet$ & $5.15[3.81,6.49]$ & 32.51 \\
\hline Overall & 42 & 44 & & $\gamma$ & $1.97[-0.90,4.84]$ & 100 \\
\hline \multicolumn{4}{|c|}{$95 \%$ Prediction interval } & & {$[-34.56,38.50]$} & \\
\hline \multicolumn{4}{|c|}{ Heterogeneity: $T^{2}=6.12, I^{2}=95.67 \%$} & & & \\
\hline \multicolumn{2}{|c|}{ Test of $\theta_{i}=\theta_{j}: Q(2)=46.24, p<0.0001$} & & Favours Control & Favours Vitamin C & & \\
\hline \multicolumn{2}{|c|}{ Test of $\theta=0: z=1.34, p=0.18$} & & & & & \\
\hline & & & $-30-20-10$ & $0510 \quad 20 \quad 30$ & & \\
\hline
\end{tabular}

E

MDA

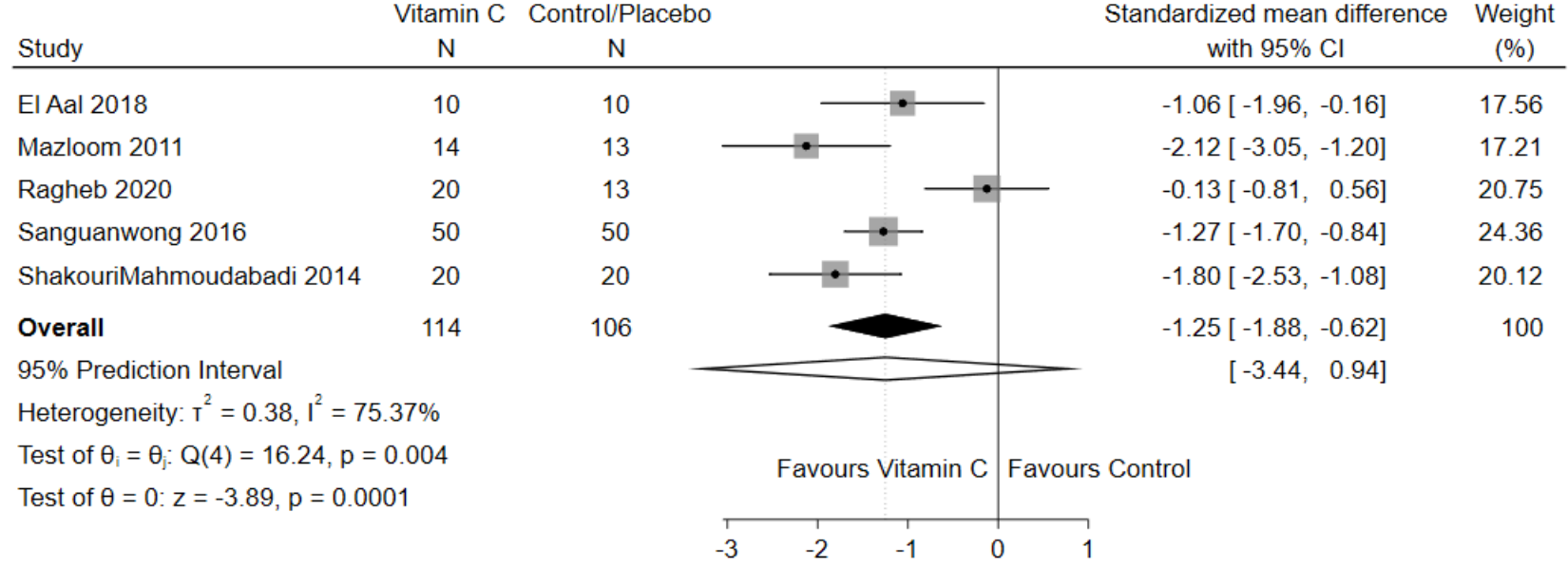

$\mathbf{F}$ 
F2-Isoprostanes

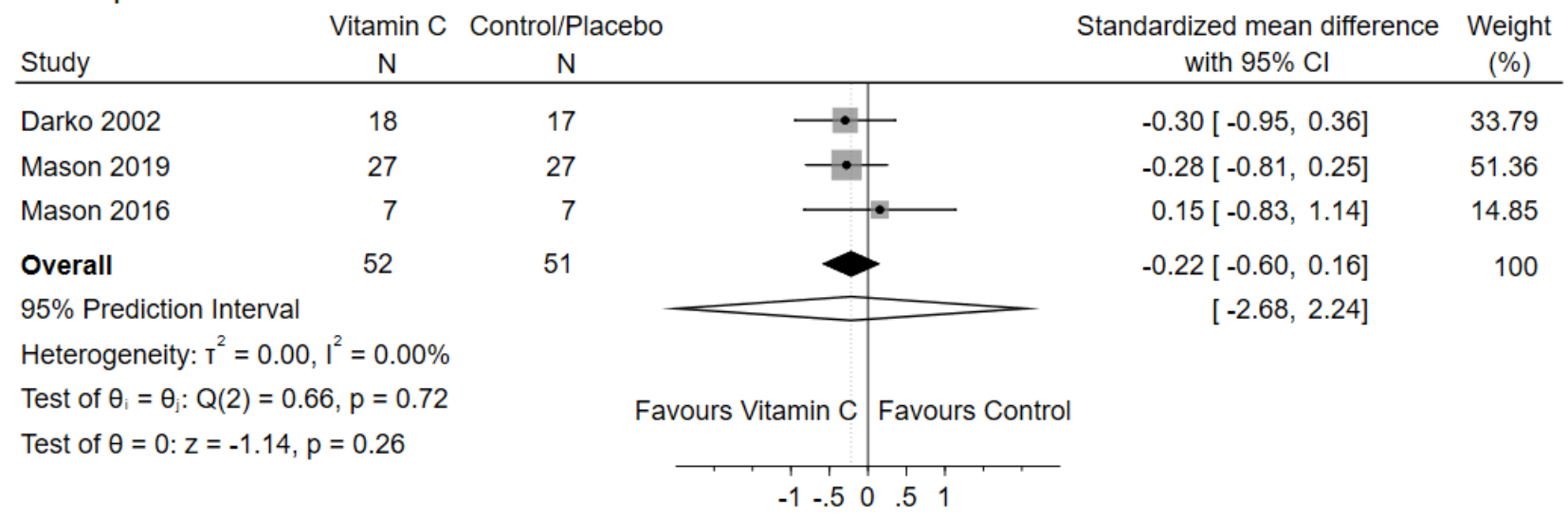

G

LDL Oxidation

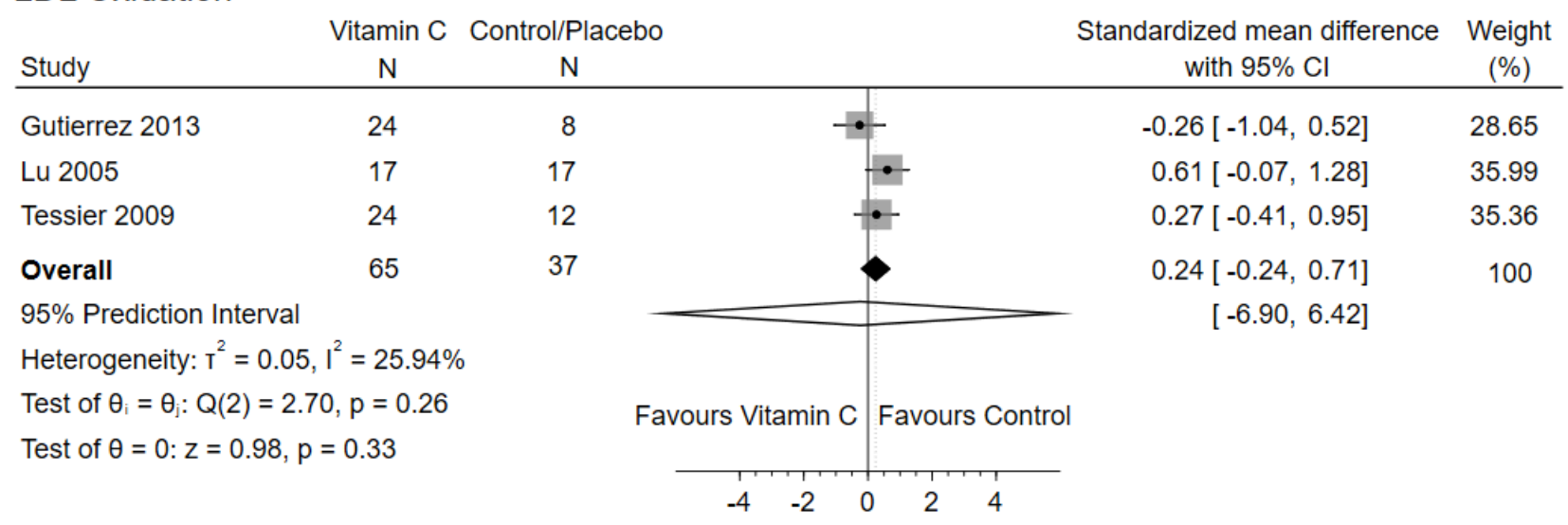

Figure S3. Forest plot of effect of vitamin C supplementation on postprandial glucose (A); fasting insulin (B); homeostasis model assessment of insulin resistance [HOMA-IR] (C); insulin sensitivity [clamp] (D); malondialdehyde (MDA) (E); F2-Isoprostanes (F); and LDL Oxidation (G). 


\section{B - Fasting glucose}
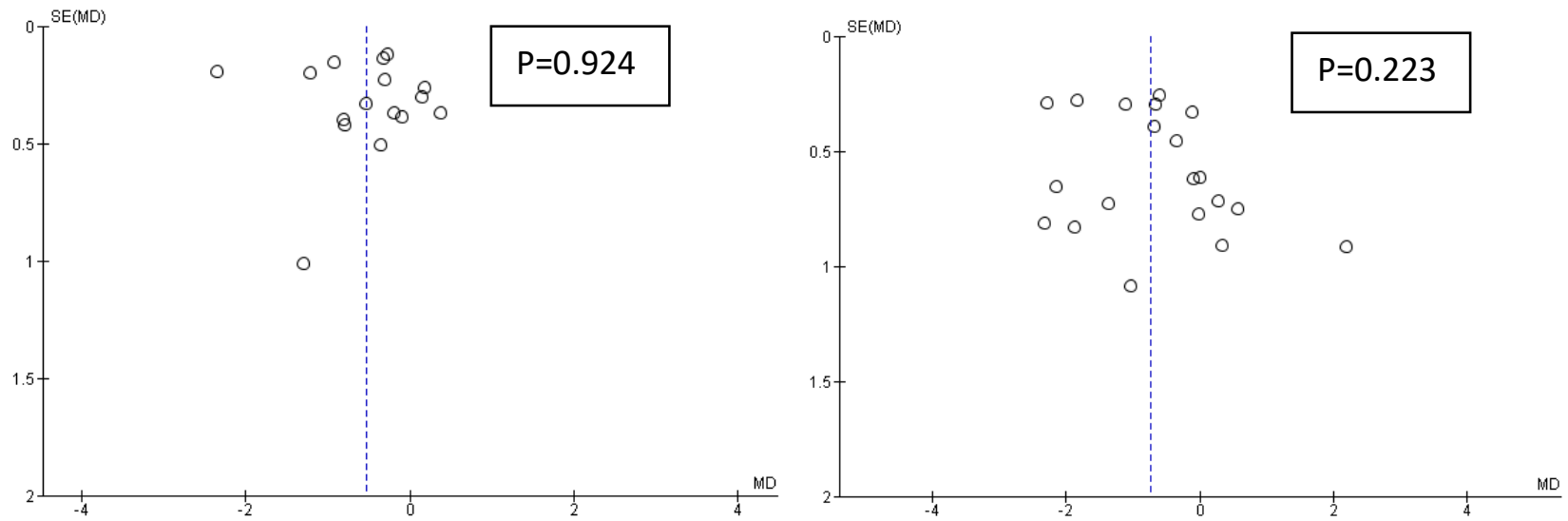

C - Triglycerides

D - Total Cholesterol
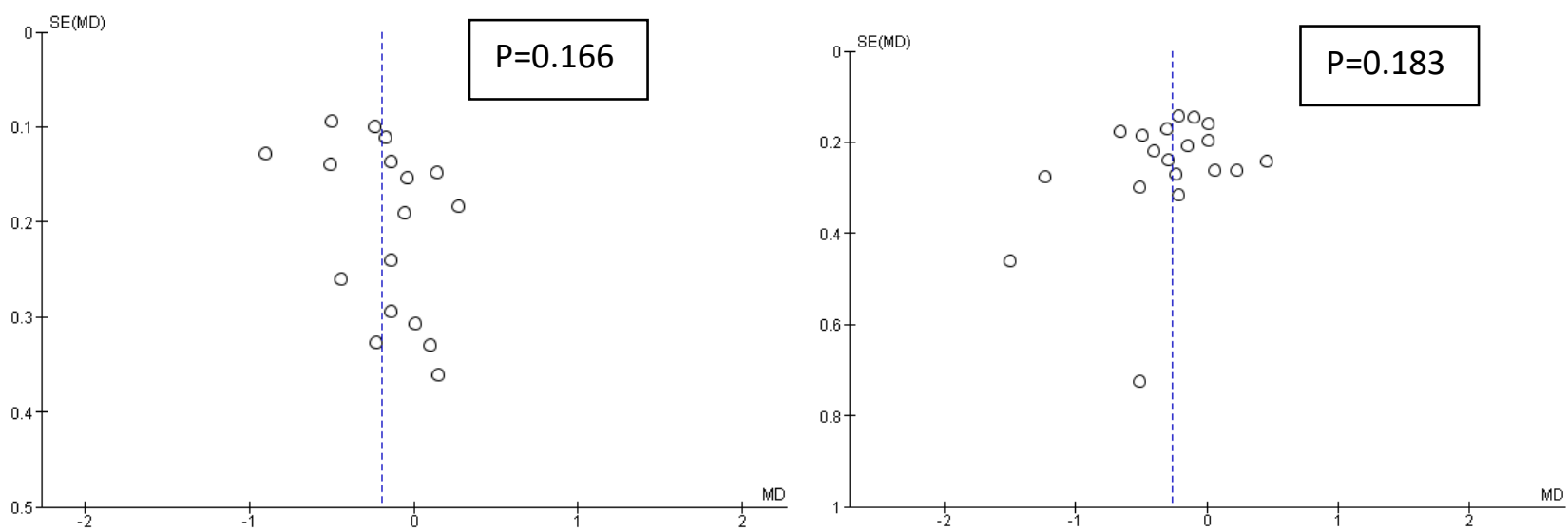

E - LDL Cholesterol

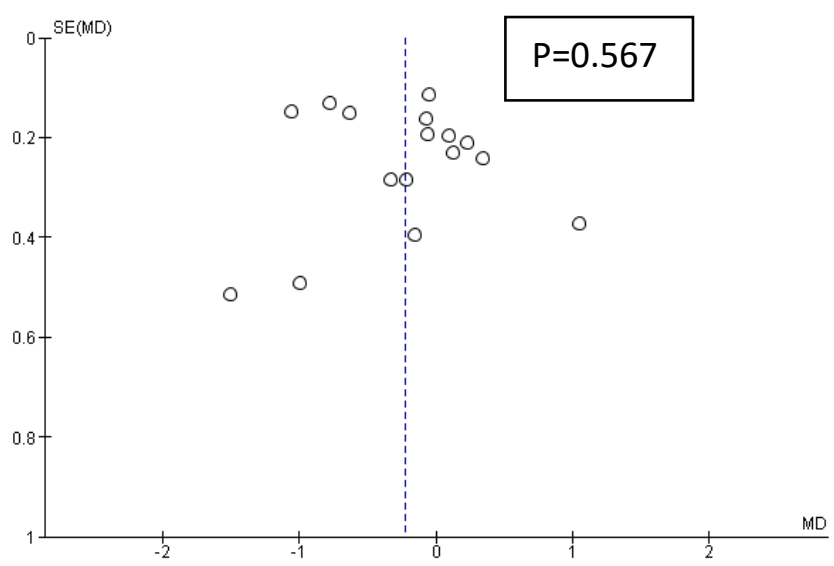

F - HDL Cholesterol

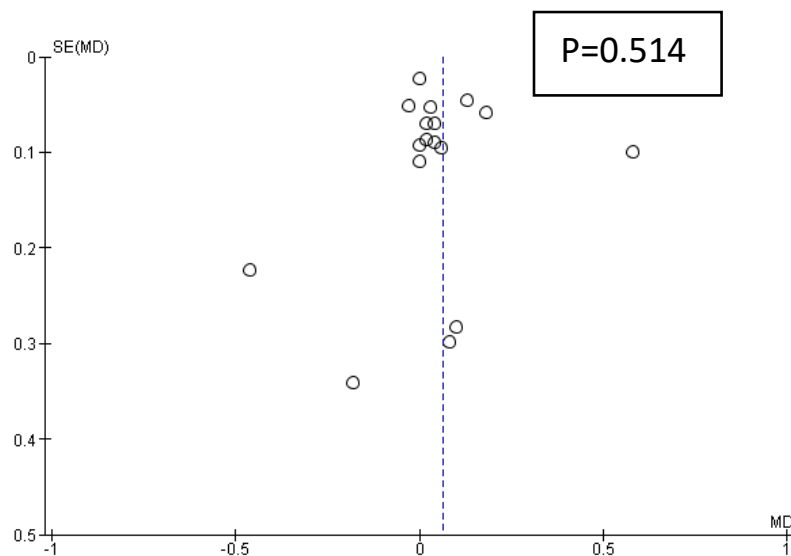

Figure S4. Publication bias funnel plots and Egger Regression significance values for outcomes with at least 10 studies and 1 medium/large size study included: HbA1c (A); fasting glucose (B); triglycerides (C); total cholesterol (D); LDL cholesterol (E); and HDL cholesterol (F). 